\title{
Three-Dimensional Carbon-coated Si/rGO Nanostructures Anchored by Nickel \\ Foam with Carbon Nanotubes for Li-ion Battery Applications
}

Jingbo Chang ${ }^{\not}$, Xingkang Huang ${ }^{\not}$, Guihua zhou, Shumao Cui, Shun Mao, Junhong Chen*

Department of Mechanical Engineering, University of Wisconsin-Milwaukee, 3200

\author{
North Cramer Street, Milwaukee, Wisconsin 53211, USA \\ E-mail: jhchen@uwm.edu \\ These authors contributed equally.
}

\begin{abstract}
A nanostructured silicon $(\mathrm{Si})$ electrode has shown great potential as a lithium-ion battery (LIB) anode because it has a charge storage capacity ten times more than that of conventional graphite; however, practical applications of Si-based anodes have been severely hindered due to their poor cyclability and low intrinsic electrical conductivity. Here we develop a novel binder-free Si-based anode through the encapsulation of $\mathrm{Si}$ nanoparticles $(\mathrm{Si} \mathrm{NPs})$ with carbon cloth and reduced graphene oxide $(\mathrm{rGO})$, where carbon nanotubes (CNTs) rooted from a nickel foam result in a strong connection mechanically and electrically between active materials and current collectors. In the resulting architecture, a dense cellular carbon cloth from carbonization of poly (methyl methacrylate) (PMMA) on $\mathrm{Si}$ surfaces can improve the electrical conductivity and accommodate the volume change, whereas rGO networks provide additional mechanical strength to maintain the integrity of electrodes. The newly designed nanostructure exhibited a high reversible capacity up to $2,700 \mathrm{mAh} \mathrm{g}^{-1}$ at $0.05 \mathrm{C}\left(130 \mathrm{~mA} \mathrm{~g}^{-1}\right)$ and $70 \%$ of capacity retention (up to $1,311 \mathrm{mAh} \mathrm{g}^{-1}$ ) at $2.6 \mathrm{~A} \mathrm{~g}^{-1}$ after 900 cycles.
\end{abstract}


Keywords: Si nanoparticles, carbon nanotubes, graphene oxide, carbonization, lithiumion battery

\section{Introduction}

Nanostructured silicon ( $\mathrm{Si}$ ) electrodes have shown great potential as lithium-ion battery (LIB) anodes. As the power demands in consumer electronics, transportation, and largescale renewable energy storage continue to increase, LIBs with a greater power and a higher energy density are urgently needed.[1-4] Si anodes[5-9] offer an alternative to commercial graphite, with Li storage capacity ten times higher than that of the graphite (372 $\mathrm{mAh} \mathrm{g}^{-1}$ ) used in commercial LIBs; thus, Si has been considered as a promising candidate for an anode material for next-generation LIBs. Unfortunately, practical applications of $\mathrm{Si}$ as an anode material are not yet a reality due to the huge volume change $(>300 \%)$ during the lithiation/delithiation process, which leads to dramatic destruction of the initial particle morphology and the loss of electrical contact between active materials and the electrode framework. In addition, the low intrinsic electrical conductivity of Si leads to dramatic pulverization of the Si anode material, rapid capacity decay, and poor cyclability, and thus should be addressed before its practical application. $[10,11]$

Significant efforts have been made towards mitigating the adverse mechanical effects of $\mathrm{Si}$ anodes and improving their overall electrochemical performance through diverse strategies, including engineering active material, current collector, binder and/or their interfaces.[12-14] A particularly attractive strategy is the use of in-situ polymerization to form a bi-functional conformal coating that binds to the Si surface and also serves as a 
short diffusion pathway for electronic conduction and three-dimensional (3D) interconnectivity. $[9,15]$ The Si nanoparticles (NPs) are trapped within the interconnected narrow pores of the polymer matrix, which can buffer the pulverization of larger particles during lithiation/delithiation and maintain good electrical connectivity among the fractured particles. Alternatively, hybrid nanostructures with carbon and various morphologies of $\mathrm{Si}$ have been created to tailor the nanostructure and buffer the mechanical strain. Nanometer-sized Si materials combined with carbon in the form of nanoparticles,[16, 17] nanowires,[18] nanotubes,[19] and core-shell nanofibers [20-22] have shown a long cycling life as candidate anode materials because they enable rapid Li transport with free volume around nanostructures and thus facilitate strain relaxation.

Carbon is a commonly used conductive additive in lithium-ion electrode materials, which can enhance electrical conductivity and constrain the large volume change of Si to maintain structural integrity by coating the Si surface with carbon.[23-27] In the electrode structure viewpoint, we adopted Si-graphene with alternating multilayered composite structures, in which Si NPs are embedded in the in-between graphene films using a dip-coating method.[28] Nanostructured $\mathrm{Si}$ with graphene coating has been proven to be one of the most suitable $\mathrm{Si}$ anode designs because the graphene can accommodate the volume expansion of Si through its void space while enhancing the electrical conductivity; $[29,30]$ however, fabricating those anodes is complex and timeconsuming because the adhesion between the active materials and current collectors is not strong enough for longer cycling. Despite these issues, we have found a safer and more economical rapid fabrication method to improve anode performance by carbonating polymer on Si NPs and anchoring active materials on current collectors by carbon 
nanotubes (CNTs). This, in turn, forms cellular carbon coating and enforces interfacial contact between active materials and substrates by CNTs.

Here we report a novel anode composed of Si NPs trapped in a 3D cellular carbon framework with reduced graphene oxide $(\mathrm{rGO})$ as the electronic fortifier. The polymer, in which Si NPs and GO sheets are well distributed, can be carbonized to become amorphous carbon on Si NPs loaded on nickel (Ni) foams; CNTs rooted from Ni forms, can help active materials stabilization during cycling of LIBs. Such an electrode offers several advantages as LIB anodes: (1) the approach opens a new pathway for scalable, low-cost, and binder-free fabrication of carbon-coated Si/rGO/CNT (SiGC) nanostructures using a viscous solution method, which can assist with the mass loading of active materials; (2) the carbonized polymer generates a dense cellular carbon on $\mathrm{Si}$ NPs/rGO surfaces and an Ni foam through the entire electrode,[31] attributing to the enhanced electrode integrity; (3) carbon coating and rGO wrapping on Si NPs restrict volume expansion and improve electrical conductivity, thereby enhancing lithium ion transport and electrical contact with Si NPs during the charge/discharge processes; and (4) the catalyzed CNT growth on Ni foams using the CVD method results in a strong interface connection, thereby helping to maintain the stability of the $\mathrm{Si}$ anode and improve its cyclability. In addition, the fabrication method using a polymer solution process leads to the uniform distribution of Si NPs and GO sheets in the carbon framework and thus high performance in cycle stability and rate capability. More importantly, CNTs grown on the Ni foam, like nails, can stabilize the active materials on the Ni foam and result in desirable structure integration. 


\section{Experimental section}

Composite solution preparation: GO sheets were produced from natural graphite powder (SP-1, Bay Carbon, MI) using the modified Hummers method ${ }^{28}$. The GO suspension (5 $\mathrm{mg} / \mathrm{ml}$ ) was prepared by dispersing the prepared GO powder into ethanol with the assistance of ultrasonication. The ratio of the PMMA to anisole was kept at $10 \%$ by dissolving $350 \mathrm{mg}$ of PMMA in $3.5 \mathrm{~g}$ of anisole. Then, Si NPs (Average particle size 50 nm, synthesized using laser from vapor phase, Alfa Aesar) were dispersed into the PMMA solution with ultrasonication. Finally, the GO suspension was mixed with Si NPs suspension, and then sonicated for $1 \mathrm{~h}$.

Growth of CNTs: A Ni foam disk (Diameter: 0.5 inch) was treated by $0.1 \mathrm{M}$ oxalic acid, dried at $80{ }^{\circ} \mathrm{C}$, and transferred into a tube furnace. CNTs were grown on the Ni foam by a chemical vapor deposition (CVD) method with a $\mathrm{C}_{2} \mathrm{H}_{2}$ flow $\left(30 \mathrm{~mL} \mathrm{~min}{ }^{-1}\right)$ at $700{ }^{\circ} \mathrm{C}$ for $20 \mathrm{~min}$.

Anode fabrication: Pretreated porous Ni foam was immersed into a suspension, and the suspension coated the $\mathrm{Ni}$ foam in the form of large-quantity, micron-sized liquid droplets that filled the Ni pores. Then, the Ni foam was removed from the suspension and dried using a nitrogen flow at $0.1 \mathrm{~L} \mathrm{~min}^{-1}$. After drying, composite films were uniformly coated on the Ni foam. To obtain the weight of active materials, the $\mathrm{Ni}$ foam was weighed before the dip-coating assembly process and after the thermal annealing process. The Si content in the SiGC electrode was defined as the weight percentage of Si NPs in the active material ( $\mathrm{Si}$ and $\mathrm{rGO})$. 
Characterization: The as-prepared samples were characterized using a Hitachi (S-4800) scanning electron microscope (SEM) equipped with a stated resolution of $1.4 \mathrm{~nm}$ operated at a $10 \mathrm{kV}$ acceleration voltage. Raman spectrum was obtained using a Renishaw 1000B Raman microscope with a 632.8-nm HeNe laser with three accumulations of 10 seconds each. Coin cells (2032-tpye) were assembled in an argonfilled glove box with the SiGC electrode and a lithium disk. Then, $1 \mathrm{M} \mathrm{LiPF}_{6}$ dissolved in ethylene carbonate/ ethyl methyl carbonate $(40: 60, \mathrm{v} / \mathrm{v})$ was employed as an electrolyte with $5 \mathrm{wt} . \%$ of fluoroethylene carbonate and $1 \mathrm{wt} . \%$ vinylene carbonate as additives. The coin cells were tested on a Land battery tester. The current density and the specific capacity were calculated based on the total mass loaded on the nickel foam. Cyclic voltammetry (CV) and electrochemical impedance spectroscopy (EIS) of the as-prepared $\mathrm{Si} / \mathrm{rGO}$ electrode were measured on a PARSTAT 4000 electrochemical station using a three-electrode cell. CV was carried out at a scanning rate of $0.05 \mathrm{mV} \mathrm{s}^{-1}$ while EIS was tested between 10,000-0.1 Hz with an amplitude of $10 \mathrm{mV}$.

\section{Result and discussion}

The fabrication process for the novel architecture of the SiGC hybrid nanostructures is schematically illustrated in Figure 1. Solution-based techniques have been proposed to fabricate 3D structures on nickel foams. The Ni foam (Figure 1a) with rooted CNTs (Figure 1b) was immersed into a well-dispersed polymer suspension to load a certain amount of suspension in the pores of the Ni foam. A mixture of Si NPs and GO sheets (Figure 1c) in poly(methyl methacrylate) (PMMA) solutions (Figure 1d) is used to form the homogeneous solution. After being lifted out of the suspension, the Ni foam was 
dried immediately using a nitrogen flow $\left(0.1 \mathrm{~L} \mathrm{~min}^{-1}\right)$, as shown in Figure 1e. The resulting nanostructures exhibited satisfactory film coverage and uniformity as expected. The sample was finally heat-treated at $700{ }^{\circ} \mathrm{C}$ for $4 \mathrm{~h}$ in a tube furnace with a flow of $\mathrm{Ar}$ $\left(0.6 \mathrm{~L} \mathrm{~min}^{-1}\right)$ and $\mathrm{H}_{2}\left(0.2 \mathrm{~L} \mathrm{~min}^{-1}\right)$ mixture to carbonate PMMA and reduce GO, thereby forming an SiGC anode as shown in Figure 1f. Due to the high viscosity of PMMA, it is easy to load massive active materials on current collectors ( $\mathrm{Ni}$ foams); therefore, no binders are necessary in this process. The as-obtained $\mathrm{Si}$ anode is a novel architecture with a frame structure in which CNTs rooted in the Ni foam stabilize the carbon-coated Si NPs/rGO hybrid nanostructures. CNTs also can enhance the electronic pathways between the active species and the main current collector at the terminal, providing both mechanical and electronic integrity of the 3D nanostructures.

To successfully confirm the designed structures, the SiGC nanostructures were characterized by scanning electron microscopy (SEM) and transmission electron microscopy (TEM). A Ni foam with an average pore size of $300 \mu \mathrm{m}$ (Figure 2a) was selected as a current collector for its interconnected network of Ni structures that allows for quick transfer of Li ions during lithiation/delithiation. CNTs grew on the Ni foam by a CVD method without an additional catalyst,[32] in which CNTs penetrated carboncoated Si NPs/rGO hybrid structures and resulted in the structural integrity of the active materials with Ni foams. The catalyzed CNTs (Figure S1) have diameters of ca. $200 \mathrm{~nm}$ and their lengths are typically several microns, where the average distance between nanotubes is about $150 \mathrm{~nm}$. The top view of the electrodes shows the Si NPs are almost covered by the rGO layers (Figure 2b). The rGO networks can be easily identified due to folds in the rGO. These unique nanostructures not only improve the electronic 
conductivity of Si NPs, but also provide void space (Figure 2c) to accommodate the volume change of $\mathrm{Si}$ NPs during lithiation, thereby maintaining excellent cycling performance in LIB applications. After carbonizing the PMMA, cellular carbon was fully coated on the Si NP surface (Figure 2d). Cross-sectional SEM images in Figure 2e and $2 \mathrm{f}$ show that CNTs tangled with rGO.

The detailed nanostructure of the carbon-coated Si NPs was further investigated using TEM (Figure 2g), indicating the Si NPs were uniformly coated with carbon. The coreshell nanocrystals were analyzed using high-resolution TEM imaging. As shown in Figure $2 \mathrm{~h}$, the amorphous carbon thickness was observed to be approximately $10 \mathrm{~nm}$. The labeled lattice spacing of $0.312 \mathrm{~nm}$ corresponds with the unique (111) plane of Si NPs.

The SEM image of an SiGC sample used for energy-dispersive X-ray spectroscopy (EDS) elemental mapping is shown in Figure 3a. The corresponding elemental mapping results are shown in Figure 3b, which confirms the presence of carbon nanotubes (red color) and the uniform distribution of Si elements (green color). The EDS plot of the SiGC nanostructures exhibits signals of carbon $\mathrm{K} \alpha(0.27 \mathrm{keV})$, oxygen $\mathrm{K} \alpha(0.53 \mathrm{keV})$, nickel $\mathrm{K} \alpha(7.4 \mathrm{keV})$, and silicon $\mathrm{K} \alpha(1.74 \mathrm{keV})$, as shown in Figure 3c. The EDS results indicate the Si content as ca. $85.2 \mathrm{wt} . \%$. The oxygen is likely from silicon oxide formed on the surface of Si NPs during the fabrication process or from residual oxygencontaining functional groups in the rGO due to the partial reduction of GO. Raman spectrum of SiGC materials, as shown in Figure 3d, exhibits a peak at ca. $520 \mathrm{~cm}^{-1}$, which is ascribed to $\mathrm{Si}$, and two peaks at 1,327 and $1,597 \mathrm{~cm}^{-1}$ that belong to a strong disorder band (D-band) and a tangential mode band (G-band) of carbon, respectively. The 
intensity of the D band is higher than that of the $\mathrm{G}$ band, indicating a large number of disordered $\mathrm{sp}^{2}$ carbons (defects) in the SiGC electrode.

The charge/discharge performance of the carbon-coated Si/rGO electrode with CNT support was evaluated using coin cells with a metallic lithium counter electrode. The specific capacity values were calculated based on the total weight of Si ( 85\% wt.\%) and carbon ( 11\% wt.\%). The initial charge (Li alloying)/ discharge (Li dealloying) capacities, as shown in Figure 4a, are approximately 3,717 and 2,735 $\mathrm{mAh} \mathrm{g}^{-1}$ at $0.05 \mathrm{C}$ rate $\left(1 \mathrm{C}\right.$-rate defined as $\left.2,600 \mathrm{~mA} \mathrm{~g}^{-1}\right)$ The initial Coulombic efficiency was then calculated to be $74 \%$. The irreversible capacity loss could be associated with the solidelectrolyte interphase (SEI) formation, the reaction of oxygen-containing functional groups on rGO or amorphous $\mathrm{C}$ from PMMA with lithium ions, and the reduction of silicon oxide on the surface of Si NPs.[29, 33-35] The reversible specific capacity of $2,700 \mathrm{mAh} \mathrm{g}^{-1}$ in our SiGC is more than six times higher than that of the theoretical capacity of graphite and seven times higher than that of commercialized highperformance graphitic anodes. When the current density increased to $260 \mathrm{~mA} \mathrm{~g}^{-1}(0.1 \mathrm{C})$, the SiGC electrode delivered ca. $2,292 \mathrm{mAh} \mathrm{g}^{-1}$ with approximately $93 \%$ capacity retention at the $50^{\text {th }}$ cycle (Figure 4b). Note that the Coulombic efficiencies are about 99\% during cycling at $0.1 \mathrm{C}$, as shown in Figure $3 \mathrm{~b}$; therefore, the SiGC displays remarkably superior capacity retention. The LIB performance of the $\mathrm{Si} / \mathrm{rGO}$ electrode without the CNTs anchored on the Ni foam was investigated (Figure S2), and showed much faster capacity fading (capacity retention of $80 \%$ after 50 cycles at $0.1 \mathrm{C}$ ). The $\mathrm{Si} / \mathrm{rGO}$ electrode with CNT support displays remarkably superior capacity retention, 
which can be attributed to the unique architecture of the SiGC electrode where CNTs can stabilize hybrid active materials on the current collectors.

The rate capability of the SiGC anodes was measured and shown in Figure 4c. The average discharge (Li dealloying) capacities are 2,010, 2,000, 1,835, 1,618, 1,321, and $830 \mathrm{mAh} \mathrm{g}^{-1}$ for $0.1 \mathrm{C}, 0.2 \mathrm{C}, 0.5 \mathrm{C}, 1 \mathrm{C}, 2 \mathrm{C}$, and $5 \mathrm{C}$, respectively. These outstanding rechargeable capacities well exemplify the high rate capability offered by these specific nanostructures. When the rate returned to $0.1 \mathrm{C}$, the capacity was recovered to 2,091 $\mathrm{mAh} \mathrm{g}^{-1}$, slightly higher than at the initial $0.1 \mathrm{C}$ rate cycle $\left(2,010 \mathrm{mAh} \mathrm{g}^{-1}\right)$ (Figure $4 \mathrm{c}$ ). This electrode was subsequently cycled at $0.5 \mathrm{C}$, showing a capacity of $1,993 \mathrm{mAh} \mathrm{g}^{-1}$ and retaining $96 \%$ capacity until the $350^{\text {th }}$ cycle. To further investigate the capacity stability, the anode was also tested at $1 \mathrm{C}$ for 900 cycles, where the capacity retained approximately $70 \%$ (up to $1,311 \mathrm{mAh} \mathrm{g}^{-1}$ ), as shown in Figure 4d.

Si-based composite electrodes recently have been investigated for long-life-cycle LIB applications.[15, 36] For example, Wu et al. used the in-situ polymerization to fabricate Si-based anodes, which demonstrated a cycle life of 5,000 cycles with more than $90 \%$ capacity retention at a current density of $6.0 \mathrm{~A} \mathrm{~g}^{-1} \cdot[15]$ Ge et al. used the etching method to form porous silicon nanowires using an HF solution, which showed the capacity remains above $1,000 \mathrm{mAh} \mathrm{g}^{-1}$ after 1,500 cycles.[37] Most recently, our group synthesized hollow Si nanostructures by templates, and the hollow Si anode delivered a capacity of $814 \mathrm{mAh} \mathrm{g}^{-1}$ at a current density of $4,800 \mathrm{~mA} \mathrm{~g}^{-1}$ and $80 \%$ retention of its reversible capacity after 700 cycles.[38] Compared with those published results, we used a simple solution method to load $\mathrm{Si}$ composites $\left(0.5 \mathrm{mg} \mathrm{cm}^{-2}\right)$ on the $\mathrm{Ni}$ foams without 
any binder, which showed that Si NPs uniformly trapped in the carbon matrices during the carbonation of polymers enforced the stability of Si NPs on current collectors. Our hybrid electrodes are comparable or superior to those reported in previous studies in terms of rate capability and cycle performance, and our method does not use any toxic acid and thus is environmentally friendly.

The excellent rate capability and cycle performance of our SiGC electrodes is likely attributed to several factors: (1) nanosized Si particles provide a high specific surface area and short pathways for lithium diffusion/reactions; (2) the interconnected rGO provides 2D network for electronic transport; (3) CNTs stabilize the active materials on the electrodes; and (4) carbonated PMMA can improve the structure stability of Si-based anodes.

The CV curve of the SiGC electrode shows two typical lithiation peaks at 0.15 and 0.26 $\mathrm{V}$, and two de-lithiation peaks at 0.34 and $0.51 \mathrm{~V}$ (Figure 5a), which is consistent with previously reported $\mathrm{Si}$ anodes.[38, 39] The EIS was characterized after charging the electrode for $4 \mathrm{~h}$ and $6 \mathrm{~h}$ at $\mathrm{C} / 10$, because the electrochemical parameters are stable at around half-charge states.[38] The Nyquist plots of the SiGC electrode at the 4 h-charged and 6 h-charged states are depicted in Figure 5b, exhibiting two semi-circuits and an inclined line, associated with the SEI layer, charge transfer, and $\mathrm{Li}^{+}$diffusion processes. The Nyquist plots were fitted with a two-constant equivalent circuit, as shown in Figure S5, and the fitting results are summarized in Table S1. The average resistances of the Ohmic, the SEI layer, the charge transfer, and the $\mathrm{Li}^{+}$diffusion are 1.9, 45.5, 4.8, and 46.5 Ohm, respectively. These resistances are greater than those of our recently reported 
multilayered $\mathrm{Si} / \mathrm{rGO}$ composite anode; $[2,28]$ the $\mathrm{Si} / \mathrm{rGO}$ composite anode shows a better rate capability, most likely because the amorphous carbon from the carbonization of the PMMA has poorer electrical conductivity than the graphene. However, the carbonized carbon works as a robust matrix and CNTs stabilize the active materials on electrodes; thus the SiCG anode exhibits a better cyclic performance compared with our previous $\mathrm{Si} / \mathrm{rGO}$ anode.[28]

To determine the stability of electrodes, we have further investigated the morphology change of the SiGC electrode by SEM characterization before and after the cycle performance test. Before cycling (Figure 6a and b), Si NPs and rGO uniformly coated on the SiGC electrode; after cycling, the 3D SiGC active material also fully coated on the current collector, where Si NPs were trapped into the rGO matrix (Figure 6c and d). Therefore, the unique structure of the electrode can effectively alleviate the pulverization and thus ensures the long cycle stability. Figure 6e shows a cross-section SEM image of an SiGC electrode. The EDS results (Figure 6f) indicated that $\mathrm{Si}$ and carbon still distributed uniformly on Ni foams after cycling. CNTs still bound active materials on current collectors, thereby stabilizing electrodes during lithiation/delithiation to achieve the excellent cycle performance of SiGC electrodes. To further investigate the stability of electrodes, the uniformity of carbon on Si NPs has been investigated by TEM after 20 cycles, which showed that carbon still uniformly coated Si NPs after cycling (Figure S6).

\section{Conclusions}

In summary, we developed binder-free Si based anodes by encapsulating Si NPs with carbon cloth and reduced graphene oxide (rGO), in which rGO networks provide lithium 
ion transport pathways, CNTs stabilize Si-based hybrid materials on anodes, and PMMA after carbonation can improve the structure stability of Si-based anodes. The fabrication method is facile, surfactant free, and environmentally-friendly. The 3D bulk electrode exhibits outstanding lithium storage performance with a high reversible specific capacity $\left(2,700 \mathrm{mAh} \mathrm{g}^{-1}\right.$ at $0.05 \mathrm{C}, 830 \mathrm{mAh} \mathrm{g}^{-1}$ at $5 \mathrm{C}$; capacity based on total weight of SiGC, respectively), high rate capability (up to $5 \mathrm{C}$ ), and superior capacity retention during cycling (70\% capacity retention with a rate of $1 \mathrm{C}$ after 900 cycles up to $1,311 \mathrm{mAh} \mathrm{g}^{-1}$ ). The SiGC electrode design is effective in mitigating detrimental effects of volume expansion/contraction and improving the electronic conductivity, charge transfer and charge diffusion during the lithiation and delithiation process, which represents a significant advancement for long-cycle Si-based materials in LIB applications.

\section{Acknowledgements}

Financial support for this work was provided by the U.S. Department of Energy (DEEE0003208) and Johnson Controls, Inc. The SEM imaging was conducted at the UWM Bioscience Electron Microscope Facility and TEM analyses were conducted in the UWM Physics HRTEM Laboratory. The authors thank Dr. H. A. Owen for technical support with SEM analysis.

\section{Appendix A. Supplementary information}

Supplementary data associated with this article can be found in the online version.

\section{References}

[1] J.B. Goodenough, Energy Environ. Sci. 7 (2014) 14-18. 
[2] V. Etacheri, R. Marom, R. Elazari, G. Salitra, D. Aurbach, Energy Environ. Sci. 4 (2011) 3243-3262.

[3] J.S. Luo, J.L. Liu, Z.Y. Zeng, C.F. Ng, L.J. Ma, H. Zhang, J.Y. Lin, Z.X. Shen, H.J. Fan, Nano Lett. 13 (2013) 6136-6143.

[4] H.G. Zhang, X.D. Yu, P.V. Braun, Nat. Nanotechnol. 6 (2011) 277-281.

[5] C.K. Chan, H.L. Peng, G. Liu, K. McIlwrath, X.F. Zhang, R.A. Huggins, Y. Cui, Nat. Nanotechnol. 3 (2008) 31-35.

[6] R.C. de Guzman, J.H. Yang, M. Ming-Cheng, S.O. Salley, K.Y.S. Ng, J. Mater. Sci. 48 (2013) 4823-4833.

[7] X.H. Liu, F.F. Fan, H. Yang, S.L. Zhang, J.Y. Huang, T. Zhu, ACS Nano 7 (2013) 1495-1503.

[8] A. Magasinski, P. Dixon, B. Hertzberg, A. Kvit, J. Ayala, G. Yushin, Nat. Mater. 9 (2010) 353-358.

[9] M.Y. Wu, J.E.C. Sabisch, X.Y. Song, A.M. Minor, V.S. Battaglia, G. Liu, Nano Lett. 13 (2013) 5397-5402.

[10] X. Su, Q.L. Wu, J.C. Li, X.C. Xiao, A. Lott, W.Q. Lu, B.W. Sheldon, J. Wu, Adv. Energy Mater. 4 (2014) 1300882

[11] D. Munao, M. Valvo, J. van Erven, E.M. Kelder, J. Hassoun, S. Panero, J. Mater. Chem. 22 (2012) 1556-1561.

[12] M.T. McDowell, S.W. Lee, W.D. Nix, Y. Cui, Adv. Mater. 25 (2013) 4966-4984.

[13] H.J. Lin, W. Weng, J. Ren, L.B. Qiu, Z.T. Zhang, P.N. Chen, X.L. Chen, J. Deng, Y.G. Wang, H.S. Peng, Adv. Mater. 26 (2014) 1217-1222. 
[14] J.Y. Ji, H.X. Ji, L.L. Zhang, X. Zhao, X. Bai, X.B. Fan, F.B. Zhang, R.S. Ruoff, Adv. Mater. 25 (2013) 4673-4677.

[15] H. Wu, G.H. Yu, L.J. Pan, N.A. Liu, M.T. McDowell, Z.A. Bao, Y. Cui, Nat. Commun., 4 (2013) 1943.

[16] Y. Wen, Y.J. Zhu, A. Langrock, A. Manivannan, S.H. Ehrman, C.S. Wang, Small 9 (2013) 2810-2816.

[17] F. Sun, K. Huang, X. Qi, T. Gao, Y.P. Liu, X.H. Zou, X.L. Wei, J.X. Zhong, Nanoscale 5 (2013) 8586-8592.

[18] T.D. Bogart, D. Oka, X.T. Lu, M. Gu, C.M. Wang, B.A. Korgel, ACS Nano 8 (2014) 915-922.

[19] H. Wu, G. Chan, J.W. Choi, I. Ryu, Y. Yao, M.T. McDowell, S.W. Lee, A. Jackson, Y. Yang, L.B. Hu, Y. Cui, Nat. Nanotechnol. 7 (2012) 309-314.

[20] M. Li, X.H. Hou, Y.J. Sha, J. Wang, S.J. Hu, X. Liu, Z.P. Shao, J. Power Sources 248 (2014) 721-728.

[21] Y. Fan, Q. Zhang, Q.Z. Xiao, X.H. Wang, K. Huang, Carbon 59 (2013) 264-269.

[22] S.A. Klankowski, R.A. Rojeski, B.A. Cruden, J.W. Liu, J. Wu, J. Li, J. Mater. Chem. A, 1 (2013) 1055-1064.

[23] S.Q. Chen, P.T. Bao, X.D. Huang, B. Sun, G.X. Wang, Nano Research 7 (2014) 8594.

[24] K. Fu, L.G. Xue, O. Yildiz, S.L. Li, H. Lee, Y. Li, G.J. Xu, L. Zhou, P.D. Bradford, X.W. Zhang, Nano Energy 2 (2013) 976-986.

[25] L.G. Xue, G.J. Xu, Y. Li, S.L. Li, K. Fu, Q. Shi, X.W. Zhang, ACS Appl. Mater. Interfaces 5 (2013) 21-25. 
[26] J.W. Wang, X.H. Liu, K.J. Zhao, A. Palmer, E. Patten, D. Burton, S.X. Mao, Z.G. Suo, J.Y. Huang, ACS Nano 6 (2012) 9158-9167.

[27] L. Yue, S.Q. Wang, X.Y. Zhao, L.Z. Zhang, J. Mater. Chem. 22 (2012) 1094-1099.

[28] J. Chang, X. Huang, G. Zhou, S. Cui, P.B. Hallac, J. Jiang, P.T. Hurley, J. Chen, Adv. Mater. 26 (2014) 758-764.

[29] J.Y. Luo, X. Zhao, J.S. Wu, H.D. Jang, H.H. Kung, J.X. Huang, J. Phys. Chem. Lett. 3 (2012) 1824-1829.

[30] L.W. Ji, H.H. Zheng, A. Ismach, Z.K. Tan, S.D. Xun, E. Lin, V. Battaglia, V. Srinivasan, Y.G. Zhang, Nano Energy 1 (2012) 164-171.

[31] D.S. Jung, T.H. Hwang, S.B. Park, J.W. Choi, Nano Lett. 13 (2013) 2092-2097.

[32] E.F. Kukovitsky, S.G. L'vov, N.A. Sainov, V.A. Shustov, Appl. Surf. Sci. 215 (2003) 201-208.

[33] S.Y. Yin, Y.Y. Zhang, J.H. Kong, C.J. Zou, C.M. Li, X.H. Lu, J. Ma, F.Y.C. Boey, X.D. Chen, ACS Nano 5 (2011) 3831-3838.

[34] Z.S. Wu, W.C. Ren, L. Wen, L.B. Gao, J.P. Zhao, Z.P. Chen, G.M. Zhou, F. Li, H.M. Cheng, ACS Nano 4 (2010) 3187-3194.

[35] B. Hertzberg, A. Alexeev, G. Yushin, J. Am. Chem. Soc. 132 (2010) 8548.

[36] Y. Yao, M.T. McDowell, I. Ryu, H. Wu, N.A. Liu, L.B. Hu, W.D. Nix, Y. Cui, Nano Lett. 11 (2011) 2949-2954.

[37] M.Y. Ge, J.P. Rong, X. Fang, C.W. Zhou, Nano Lett. 12 (2012) 2318-2323.

[38] X. Huang, J. Yang, S. Mao, J. Chang, P.B. Hallac, C.R. Fell, B. Metz, J. Jiang, P.T. Hurley, J. Chen, Adv. Mater. 26 (2014) 4326-4332. 
[39] X. Huang, H. Pu, J. Chang, S. Cui, P.B. Hallac, J. Jiang, P.T. Hurley, J. Chen, ACS Appl. Mater. Interfaces 5 (2013) 11965-11970. 


\section{Figure Captions}

Figure 1. Schematic of the fabrication process for SiGC hybrid nanostructures. (a) A nickel foam substrate. (b) CVD growth of CNTs on nickel foams. (c) Mixture of Si NPs and GO suspension in ethanol. (d) Addition of PMMA into the mixed solution. (e) Deposition of GO/Si/PMMA mixtures onto the CNT-coated nickel foam and (f) thermal annealing at $700{ }^{\circ} \mathrm{C}$ for $4 \mathrm{~h}$.

Figure 2. An SiGC electrode. (a) CNT/3D porous Ni foam electrode coated with carboncoated Si/rGO nanostructures. Top view: (b) carbon-coated Si NPs in rGO networks; (c) carbon-coated Si NPs; (d) high-magnification SEM image of carbon-coated Si NPs. Cross-sectional view: (e) SEM image and (f) higher magnification image of the penetration of carbon-coated Si NPs/rGO nanostructures by CNTs. TEM images of (g) carbon-coated Si NPs; (h) HRTEM image of the carbon-coated Si NPs.

Figure 3. (a) SEM image of carbon-coated Si/rGO electrodes, (b) EDS elemental mapping of overlapping Si and C, and (c) EDS plot of the electrode. (d) Raman spectrum of the electrode.

Figure 4. Electrochemical characteristics of the SiGC electrode. (a) Galvanostatic charge/discharge profiles of the first two cycles at 0.05 C-rate, (b) capacity retention and Coulombic efficiency at $0.05 \mathrm{C}$-rate for the first two cycles and then $0.1 \mathrm{C}$ for 50 cycles. (c) Rate capability and (d) long-term cyclic performance at $1 \mathrm{C}$. 
Figure 5. (a) CV curve and (b) Nyquist plots at various charge states of the SiGC electrode, where discrete points and solid lines represent the experimental data and the fitting results, respectively.

Figure 6. SEM images of SiGC electrodes before (a and b) and after (c and d) 900 cycles at a current rate of $1 \mathrm{C}$. Cross-section view SEM image (e) and the corresponding EDS elemental mapping (f) of SiGC electrodes after cycling. 


\section{Figure 1}

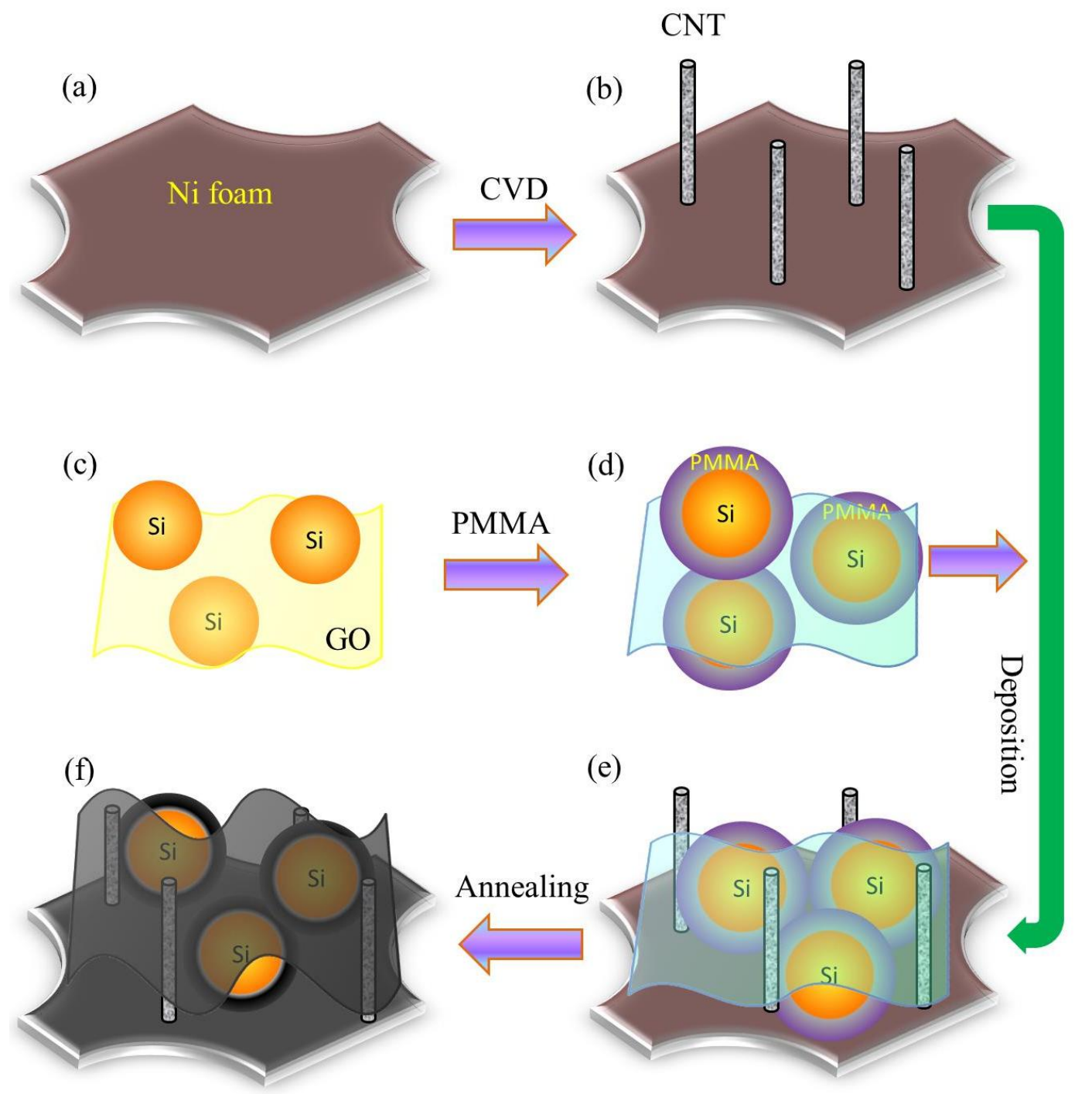


Figure 2

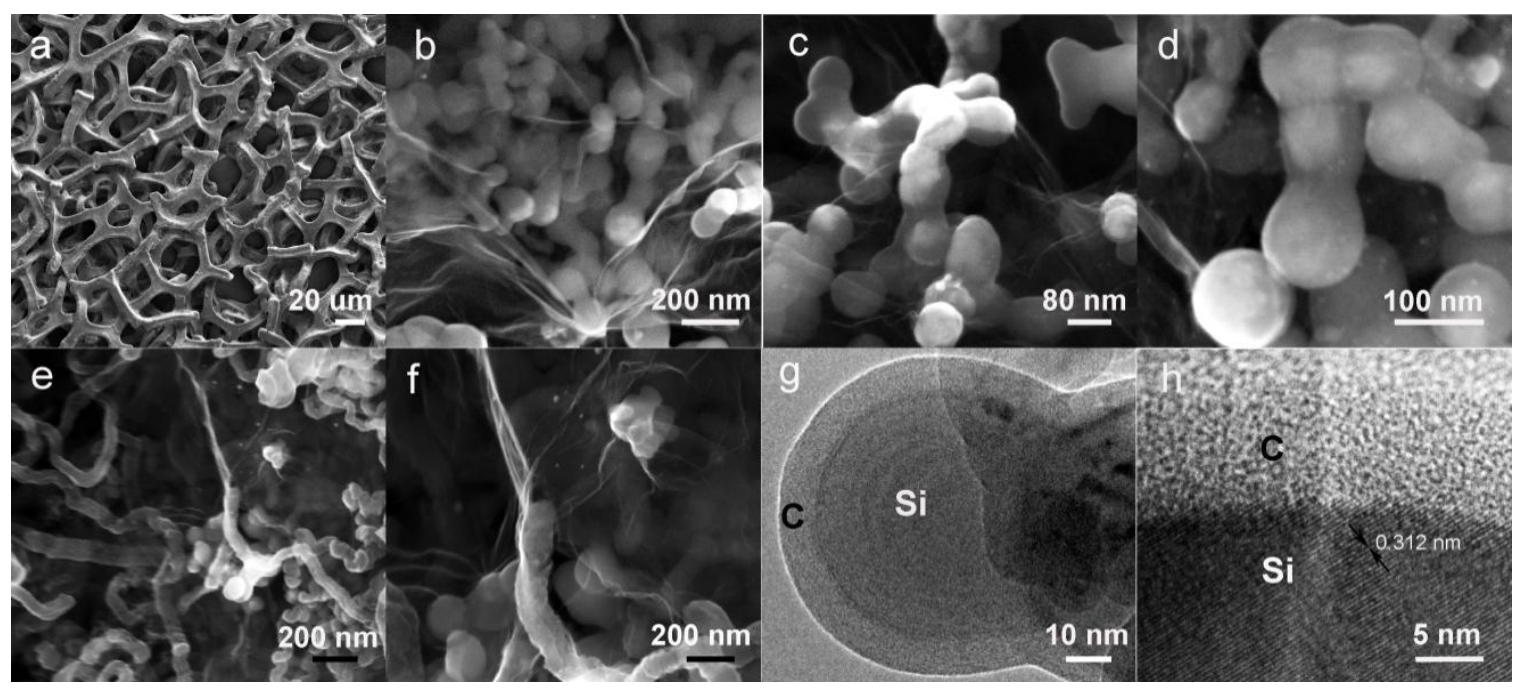


Figure 3
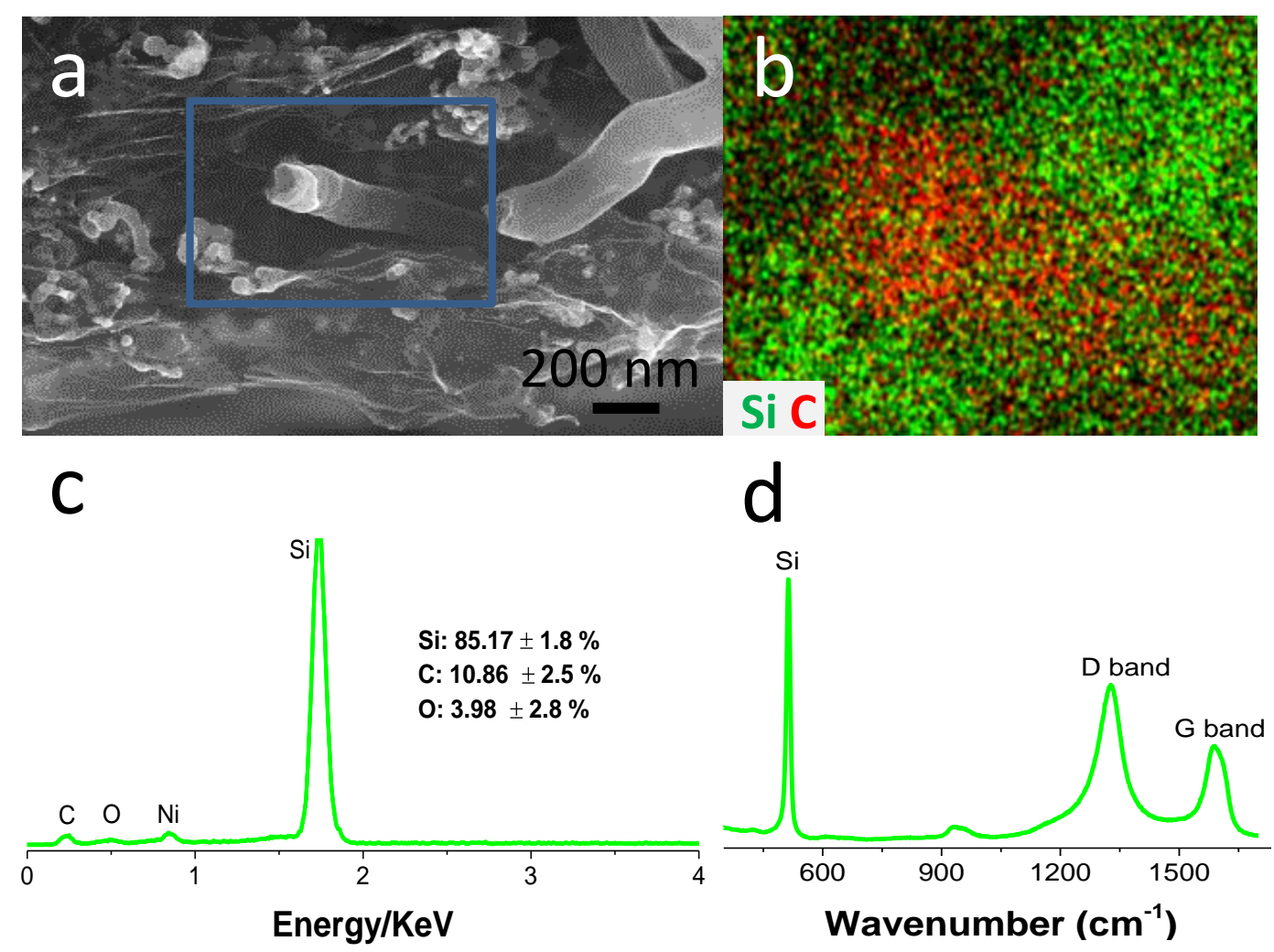


\section{Figure 4}
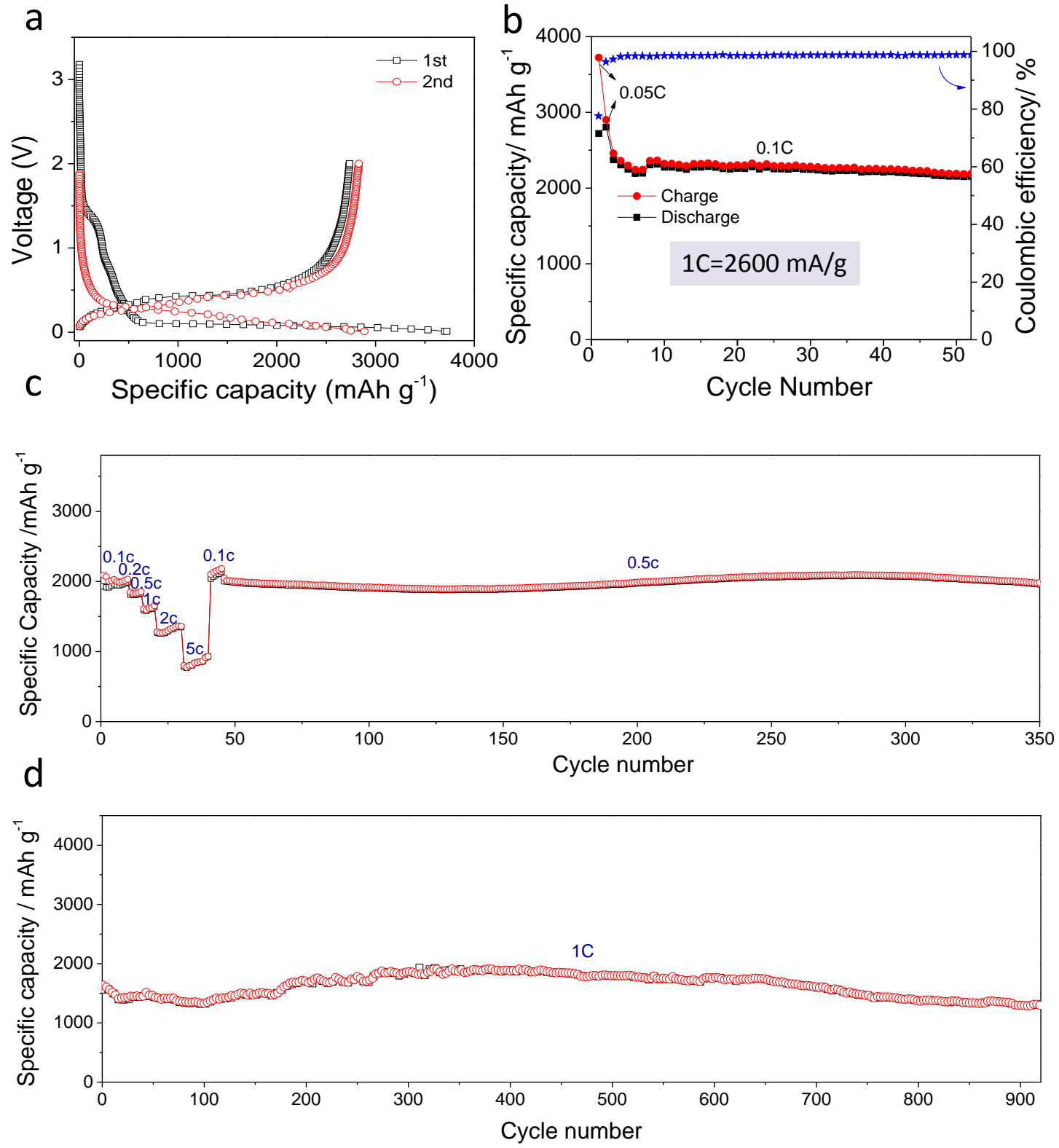
Figure 5
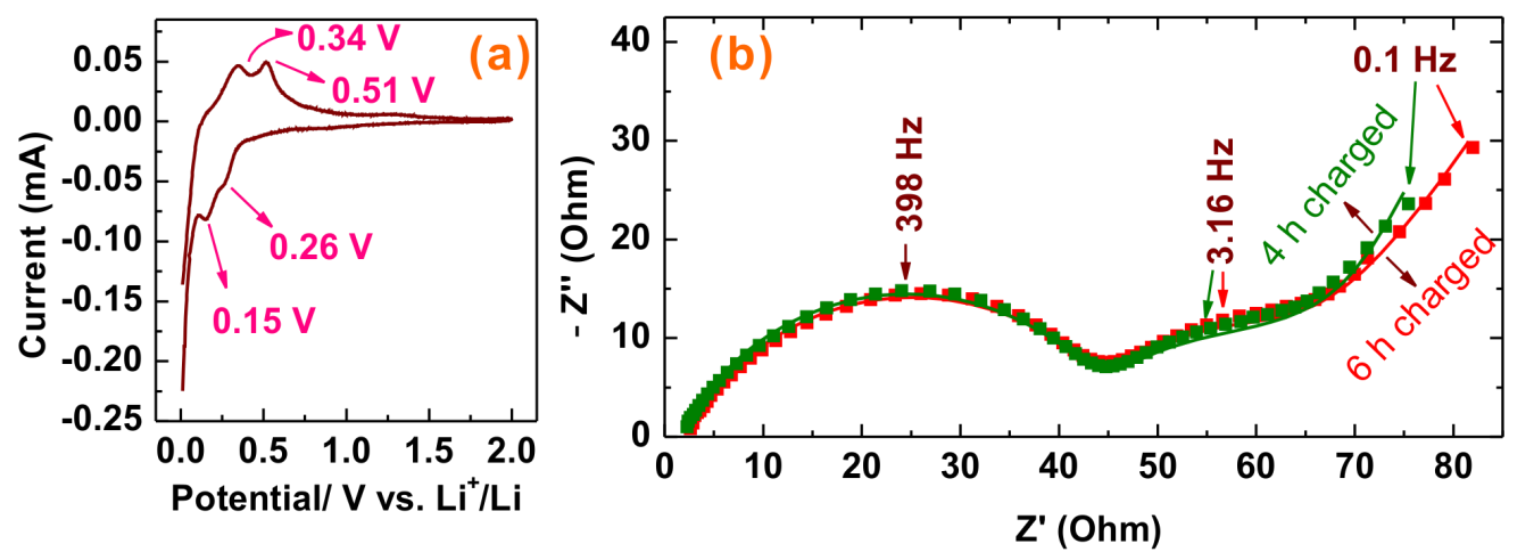
Figure 6

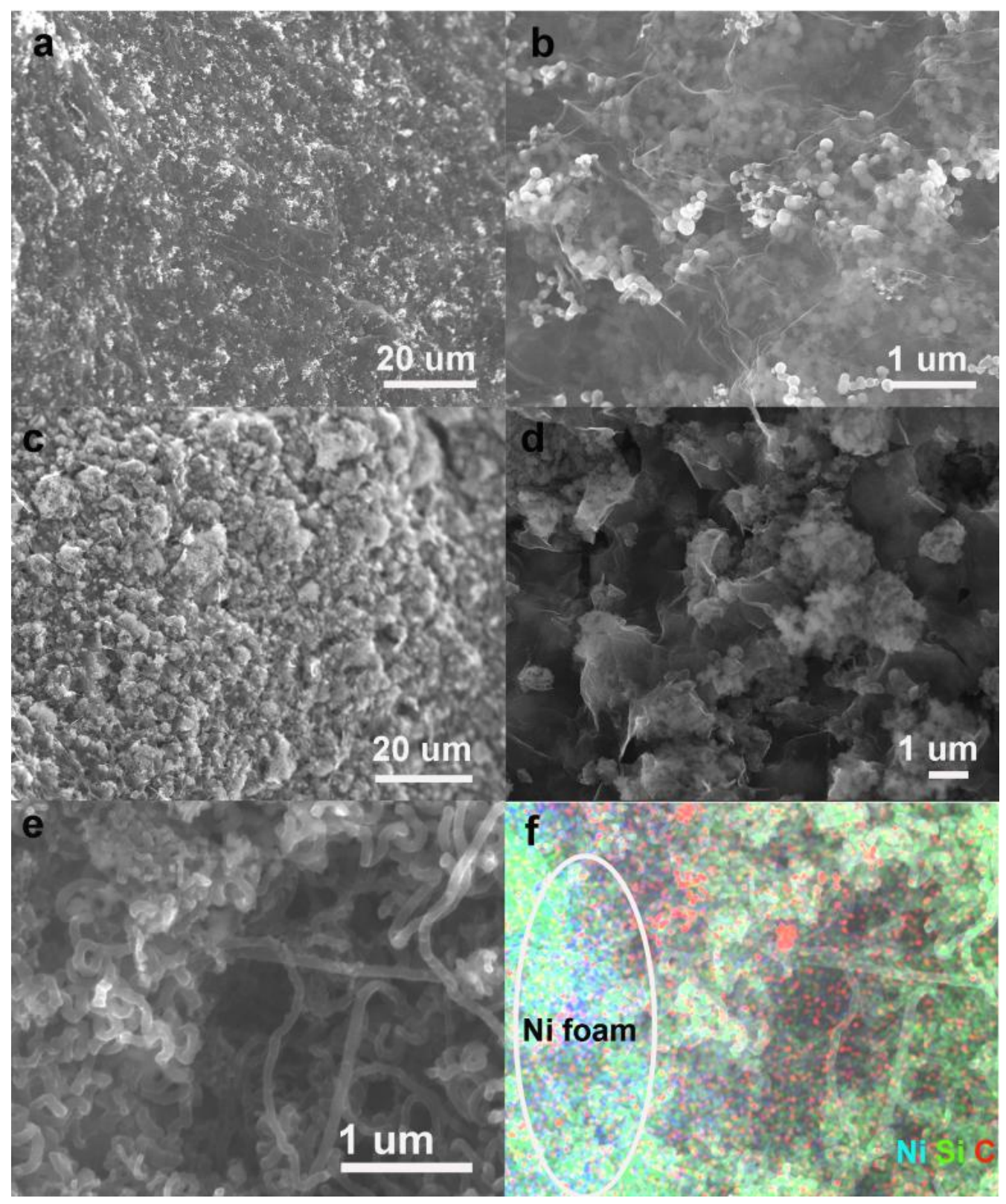




\section{Biosketch and Photograph}

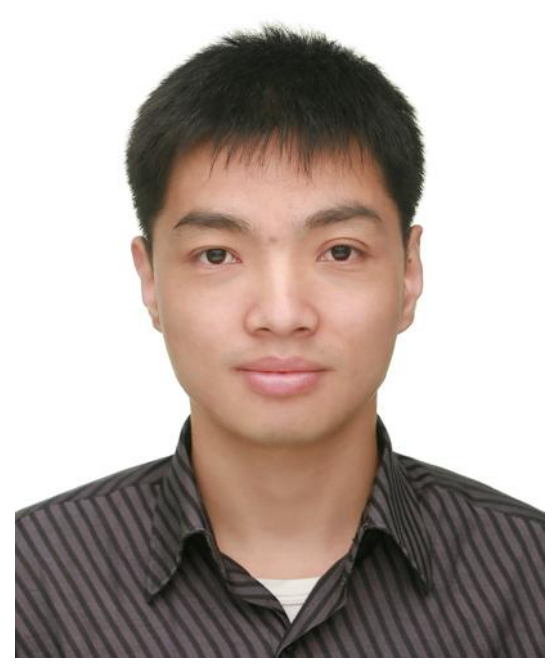

Jingbo Chang received his M.S. degree in condensed matter physics from Lanzhou University in 2006 and Ph.D. in applied chemistry from Ajou University in 2011. He has been a postdoctoral fellow at the University of Wisconsin-Milwaukee (UWM) since 2012. His research interests mainly focus on surface modification, novel structure design, and applications of nanomaterials, including lithium-ion batteries for energy storage as well as chemical sensors for detection of heavy metal ions in drinking water.

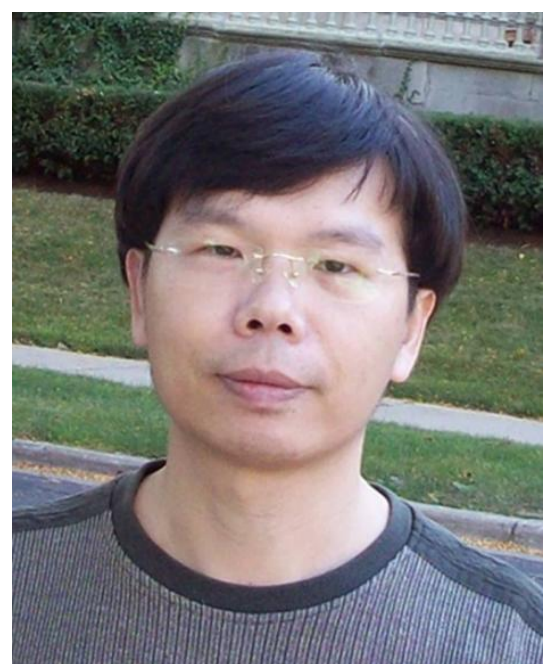

Xingkang Huang received his $\mathrm{Ph} . \mathrm{D}$. from Department of Chemistry at Xiamen University, China in 2007. His research interests focus on anode and cathode materials for lithium-ion batteries. 


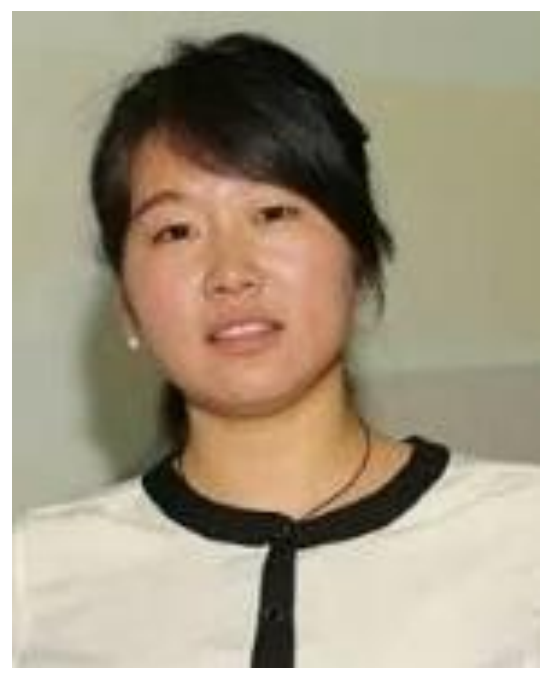

Guihua Zhou is a Ph.D. student in the Department of Mechanical Engineering at the University of Wisconsin-Milwaukee. Her current research focuses on the real-time detection of contaminants (e.g., E. coli and heavy metal ions) in water with a graphenebased sensor platform.

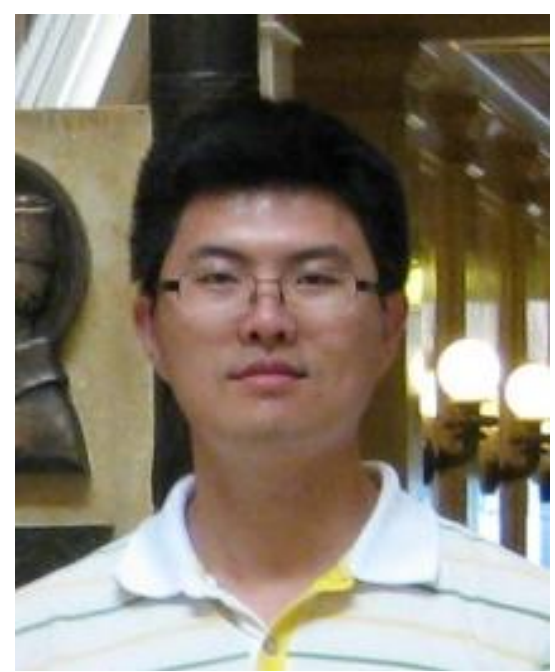

Shumao Cui received his Ph.D. in Mechanical Engineering from the University of Wisconsin-Milwaukee (UWM) in 2013 and is currently a postdoctoral fellow at UWM. His research interests include the synthesis of nanocrystals, nanohybrids combining nanocarbons (graphene and CNTs) with nanocrystals, novel two-dimensional (2D) nanomaterials, and their environmental and energy applications for chemical sensors, lithium-ion batteries, and supercapacitors. 


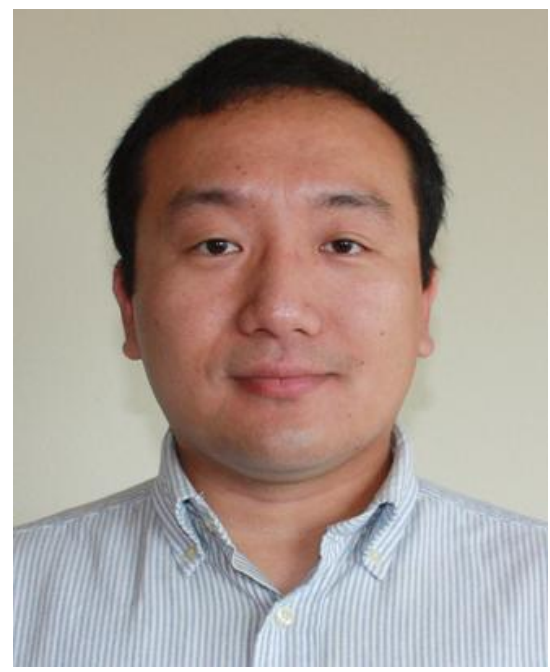

Shun Mao received his Ph.D. in Mechanical Engineering from the University of Wisconsin-Milwaukee in 2010 for the study of hybrid nanomaterial-based electronic biosensors. He is currently a postdoctoral fellow at UWM. His research focuses on hybrid 2D/0D nanomaterials for environmental and energy applications.

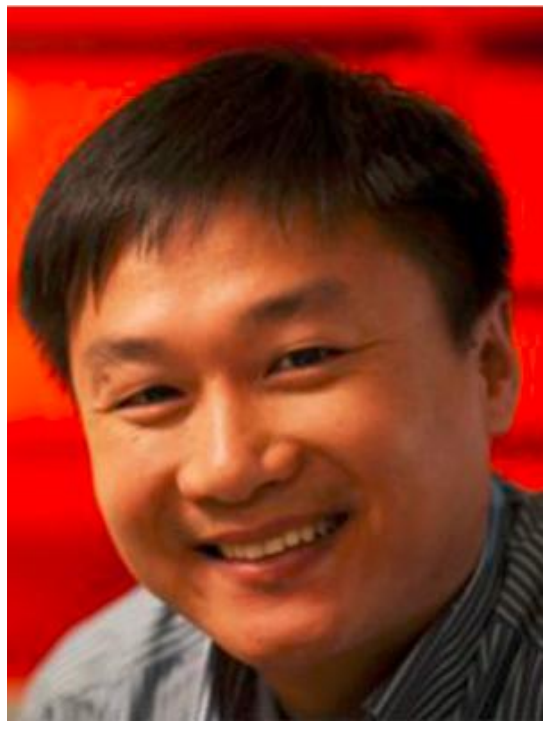

Junhong Chen is a Professor of Mechanical Engineering and a Professor of Materials Science and Engineering at UWM. His research interests lie in nanomaterial innovation for sustainable energy and environment (http:// www.uwm.edu/nsee/). 
Three-Dimensional Carbon-coated Si/rGO Nanostructures Anchored by Nickel Foam with Carbon Nanotubes for Li-ion Battery Applications

Jingbo Chang* Xingkang Huang* ${ }^{*}$, Guihua zhou, Shumao Cui, Shun Mao, Junhong Chen*

Department of Mechanical Engineering, University of Wisconsin-Milwaukee, 3200 North

Cramer Street, Milwaukee, Wisconsin 53211, USA

E-mail: jhchen@uwm.edu

Table of Contents

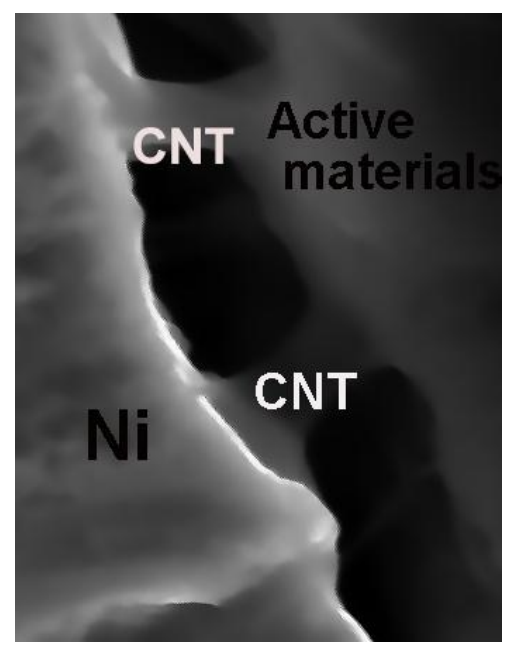

A novel binder-free Si-based anode has been developed on nickel (Ni) foam through the encapsulation of Si nanoparticles ( $\mathrm{Si}$ NPs) with carbon cloth and reduced graphene oxide (rGO), where carbon nanotubes (CNTs) rooted from the $\mathrm{Ni}$ foam results in a strong connection mechanically and electrically between active materials and current collectors. 